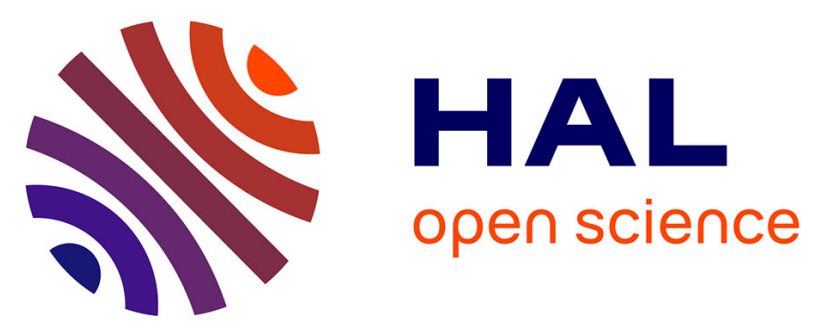

\title{
3D model related to the publication: A pangolin (Manidae, Pholidota, Mammalia) from the French Quercy phosphorites (Pech du Fraysse, Saint-Projet, Tarn-et-Garonne, late Oligocene, MP 28) \\ Jean-Yves Crochet, Lionel Hautier, Thomas Lehmann
}

\section{To cite this version:}

Jean-Yves Crochet, Lionel Hautier, Thomas Lehmann. 3D model related to the publication: A pangolin (Manidae, Pholidota, Mammalia) from the French Quercy phosphorites (Pech du Fraysse, Saint-Projet, Tarn-et-Garonne, late Oligocene, MP 28). MorphoMuseum, 2015, 1 (3), pp.e1. 10.18563/m3.1.3.e1 . hal-01923071

\section{HAL Id: hal-01923071 \\ https://hal.science/hal-01923071}

Submitted on 9 Nov 2020

HAL is a multi-disciplinary open access archive for the deposit and dissemination of scientific research documents, whether they are published or not. The documents may come from teaching and research institutions in France or abroad, or from public or private research centers.
L'archive ouverte pluridisciplinaire HAL, est destinée au dépôt et à la diffusion de documents scientifiques de niveau recherche, publiés ou non, émanant des établissements d'enseignement et de recherche français ou étrangers, des laboratoires publics ou privés. 


\section{D model related to the publication: A pangolin (Manidae, Pholidota, Mammalia) from the French Quercy phosphorites (Pech du Fraysse, Saint-Projet, Tarn-et- Garonne, late Oligocene, MP 28)}

\section{CROCHET J.-Y.a, HAUTIER L. ${ }^{a^{*}}$ and LEHMANN T. ${ }^{\mathrm{b}}$}

a Institut des Sciences de l'Evolution de Montpellier, Université Montpellier 2, CNRS, IRD, Cc 064; place Eugène Bataillon, 34095 Montpellier Cedex 5, France

${ }^{b}$ Senckenberg Research Institute and Natural History Museum Frankfurt, Palaeoanthropology and Messel Research Department, Senckenberganlage 25, 60325 Frankfurt am Main, Germany

* corresponding author: Lionel.Hautier@univ-montp2.fr

Abstract: This contribution contains the 3D model described and figured in the following publication: Crochet, J.-Y., Hautier, L., Lehmann, T., 2015. A pangolin (Manidae, Pholidota, Mammalia) from the French Quercy phosphorites (Pech du Fraysse, SaintProjet, Tarn-et-Garonne, late Oligocene, MP 28). Palaeovertebrata 39(2)-e4. doi: 10.18563/pv.39.2.e4

Key words: Oligocene, Pangolin, Pech du Fraysse, Quercy Phosphorites

Submitted 13.01.2015, Accepted 14.09.2015. doi: 10.18563/m3.1.3.e1

(C) Copyright Lionel Hautier September 2015

\section{TECHNICAL AND SPECIMEN-RELATED PARAMETERS}

\begin{tabular}{|c|c|}
\hline Specimen inventory number & UM PFY 4051 \\
\hline Species & Necromanis cf. franconica \\
\hline Repository institution & Université de Montpellier 2 \\
\hline 3D data acquisition institution & ISE-M, Université de Montpellier \\
\hline 3D data acquisition method & $\mathrm{X}$-ray $\mu \mathrm{CT}$ \\
\hline 3D data acquisition facility model & In Vivo Mictotomograph SkyScan 1076 \\
\hline 3D data acquisition operator & Renaud Lebrun \\
\hline Voxel size of original dataset & $0.144 \mathrm{~mm}$ \\
\hline Author of derived 3D surface model & Lionel Hautier \\
\hline Model ID & M3\#12 UM PFY 4051 \\
\hline Model short description & $\begin{array}{l}\text { A partial left humerus from Pech du Fraysse } \\
\text { (Saint-Projet, Tarn-et-Garonne, France), MP } 28 \\
\text { (late Oligocene) }\end{array}$ \\
\hline
\end{tabular}

\section{METHODS}

AVIZO 7.1 (Visualization Sciences Group) software was used for segmentation. The humerus was extracted within a "labelfield" module of AVIZO, using the segmentation threshold selection tool. The 3D model is provided in .ply format, and as such can be opened with a wide range of freeware. Additional files specific to ISE-MeshTools (Lebrun, 2014) are provided in order to visualize the innominate in standard orientation.

\section{ACKNOWLEDGEMENTS}

Data presented in this work were produced through the technical facilities of the MRI platform and of the labEx CeMEB. This is
ISE-M contribution 2015-172

\section{BIBLIOGRAPHY}

Crochet, J.-Y., Hautier, L., Lehmann, T., 2015. A pangolin (Manidae, Pholidota, Mammalia) from the French Quercy phosphorites (Pech du Fraysse, SaintProjet, Tarn-et-Garonne, late Oligocene, MP 28). Palaeovertebrata 39 (2)-e4. doi: 10.18563/pv.39.2.e4

Lebrun, R., 2014. ISE-MeshTools, a 3D interactive fossil reconstruction freeware. 12th Annual Meeting of EAVP, Torino, Italy. 